\title{
ADSORPTION OF THIOCYANATE TO THE PLASMA PROTEINS*

\author{
WITH SPECIAL REFERENCE TO THE DISTRIBUTION OF THIS \\ ION BETWEEN AQUEOUS HUMOUR AND PLASMA
}

BY

\section{HUGH DAVSON}

Medical Research Council, Department of Physiology, University College, London

KinSEY AND PALM (1955) have described the kinetics of penetration of two ions, sodium and thiocyanate, into the aqueous humour, and have concluded that there is a fundamental difference in their modes of penetration; the thiocyanate ion, contrary to an original claim (Kinsey and Grant, 1942), penetrating by "diffusion", whilst sodium is said to penetrate by " secretion". An inspection of their results shows that this difference really depends largely on the different steady-state distributions between plasma and aqueous humour that are apparently reached by the two ions, sodium giving a ratio: Concentration in Aqueous Humour/Concentration in Plasma of approximately unity, whilst thiocyanate gave a ratio of 0.75 . The low steady-state ratio shown by thiocyanate would, in the past, have been attributed to the circumstance that this ion is partly adsorbed to the plasma proteins, so that only 75 per cent. would be freely diffusible; the distribution-ratio of the diffusible fraction would, therefore, have been considered as approximately unity, like that of sodium (Kinsey and Grant, 1942, Davson, DukeElder, Maurice, Ross, and Woodin, 1949; Davson and Matchett, 1953).

However, Kinsey and Palm now state that this adsorption of thiocyanate to the plasma proteins is only apparent, resulting from the presence of some other material, with chemical reactions similar to those of thiocyanate, that is adsorbed or chemically united to the plasma proteins. This claim encounters serious theoretical difficulties in the realm of colloid chemistry. The thiocyanate ion lies at the extreme end of the lyotropic series of anions, which means that its power of hydrating colloids is greater than that of iodide, bromide, and chloride, in that order. To increase the hydration of a colloid, it is generally considered necessary that the ion doing so should be adsorbed to the colloidal particles, thereby carrying its own shell of orientated water-dipoles with it. Thus, if Kinsey and Palm's claim could be substantiated, this would demand a complete re-interpretation of lyotropic phenomena; if, on the other hand, it is incorrect, we should expect that, on dialysing plasma against solutions of sodium chloride, bromide, iodide, and thiocyanate, the distribution-ratios: Concentration in Dialysate/Concentration in Plasma would decrease in this order. The distributions of chloride, bromide, and iodide have already been determined (Davson, 1955) by

*Received for publication June 28, 1955. 
techniques that are unlikely to give artefacts (chemical analysis of chloride; isotopic analysis of bromide and iodide). The ratios are as follows:

\section{Chloride 1.04 Bromide 0.96 Iodide 0.85}

Thus, if thiocyanate did, indeed, give a dialysis-ratio of 0.75 or thereabouts, it would adopt its natural place in the lyotropic series.

It is easy, however, to submit the matter to a far more stringent test. Fig. 1 (a) represents schematically the result of dialysing plasma against a saline solution containing thiocyanate, the final concentration in the outside medium being arbitrarily indicated as 75 units per litre. The black block indicates the concentration of non-diffusible material in the plasma that gives the same chemical reaction as thiocyanate, and amounts to 25 units per litre. The white blocks indicate the concentrations of diffusible material in plasma and aqueous humour, namely 75 units per litre. The experimentally determined dialysis-ratio will thus be $75 / 100=0 \cdot 75$; if the black block indicated non-thiocyanate artefact material, we could conclude that no thiocyanate was adsorbed to the proteins of plasma, and that the distribution-ratio for the thiocyanate ion was unity, namely, 75/75.

If this were true, we could predict with certainty the effect of dialysing the plasma against a thiocyanate solution ten times stronger. Thus, the amount of artefact material cannot increase, and will remain the same at 25 units per litre; the remaining concentrations will increase to 750 units per litre as in Fig. $1(b)$ and the dialysis-ratio will be $750 / 775=0.965$.

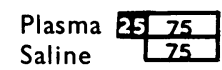

(a) $r=\frac{75}{100}=0.75$

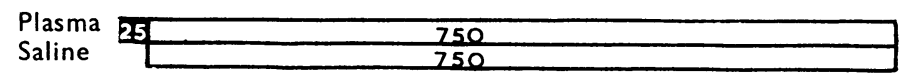

(b) $r=\frac{750}{755}=0.965$

FIG. 1 (a).-Results of dialysis of saline against plasma. Concentration of thiocyanate 75 units per litre. In plasma there is an additional 25 units per litre which may be either adsorbed thiocyanate or artefact material.

FIG. 1 (b).-Results of dialysis with solution of ten times concentration. If the additional material is artefact, the new ratio will be $750 / 775=0.965$.

If, on the other hand, the extra material in the plasma really is thiocyanate, then increasing the concentration of thiocyanate in the plasma will increase the amount bound to the protein, not necessarily by a factor of 10 , since adsorption is not a linear function of concentration, but certainly by a factor close to this; the position will then be approximately represented by Fig. 1(c),

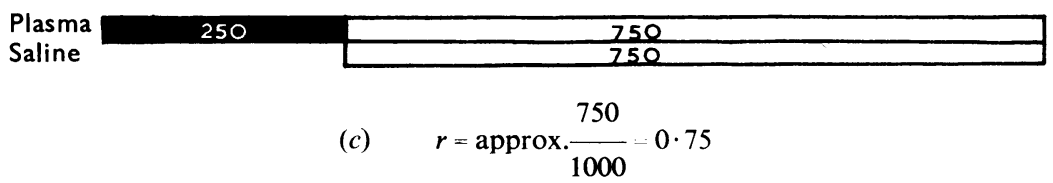

FIG. $1(c)$.-Same as $(b)$, but on the assumption that the additional material is adsorbed thiocyanate, in which case it now becomes about ten times as mucn, giving ratio of about $750 / 1,000=0 \cdot 75$. 
and the dialysis-ratio will be fairly close to the original value of $0 \cdot 75$.

The results of an experiment in which the same sample of plasma was dialysed against steadily increasing concentrations of thiocyanate in isotonic saline are as follows:

$\begin{array}{lllll}\text { Final Concentration in Saline }(m M): & 0.405 & 1.12 & 2.83 & 6.35 \\ \text { Dialysis-Ratio: } & 0.73 & 0.74 & 0.75 & 0.795\end{array}$

Thus the dialysis-ratio has changed only from 0.73 to 0.795 on increasing the thiocyanate concentration by a factor of about 15 , a result consistent with the theory of adsorption, but completely incompatible with Kinsey and Palm's assumption.

On decreasing the concentration of thiocyanate from some arbitrarily chosen value by a factor of 10 , the dialysis-ratio should change from 0.75 to 0.23 on the basis of the " artefact theory"; the results of a second experiment, in which the concentration was reduced by more than a factor of 10 , are as follows:

$\begin{array}{llllll}\text { Final Concentration in Saline }(m M): & 0.67 & 0.375 & 0.23 & 0.12 & 0.04 \\ \text { Dialysis-Ratio: } & 0.76 & 0.70 & 0.715 & 0.675 & 0.595\end{array}$

Once again, the results are consistent with the theory of adsorption, but quite incompatible with the artefact theory.

A final proof that the observed dialysis-ratios are not artefacts, may be provided by examining the actual concentration of thiocyanate-reacting material in the plasma before dialysing it against a thiocyanate solution; the concentration of artefact in the plasma clearly cannot be greater than this. In the first experiment, quoted above, the concentration was $0.064 \mathrm{mM}$; if we make the generous concession that all this material is artefact material, then the dialysis-ratio to be expected on the assumption that there is no adsorption is $6 \cdot 35 / 6 \cdot 414=0.99$ when the plasma is dialysed against the strongest solution, whereas in fact it was 0.795 . In the second experiment the initial plasma concentration was much lower, $0.01 \mathrm{mM}$; on the same assumption the dialysis-ratio should be $0.67 / 0.68=0.985$, whereas it was $0 \cdot 76$.

It is therefore quite clear that the thiocyanate ion is, indeed, bound by adsorptive forces to the plasma proteins, and that the proportion so bound is of the order of 25 per cent. Consequently, if we accept the experimental findings of Kinsey and Palm, to the effect that the distribution-ratio between plasma and aqueous humour is $\mathbf{0 . 7 5}$, this must mean that the distribution ratio for the diffusible fraction is approximately unity. One may conclude tentatively that there is probably little in the kinetic studies described by Kinsey and Palm to suggest that the modes of penetration of the two ions, sodium and thiocyanate, are not fundamentally the same. This does not, of course, mean that they are identical, but simply that present techniques of study have not progressed to the point where subtle differences may be revealed by kinetic studies of this sort; it is likely that any differences in 
behaviour that can be demonstrated will be attributable to the different fates of the ions when they have penetrated the blood-aqueous barrier. Thus, the thiocyanate ion is taken up to quite a considerable extent by the lens (Davson, 1955), whilst the sodium ion is not taken up (Langham and Davson, 1949) to anything like the same degree.

Therefore, until the experimental results of Kinsey and Palm have been re-computed on the basis of the present findings, and submitted to a statistical analysis (the greater scatter in the results for thiocyanate will make this necessary now that the steady-state distribution-ratio for this ion approximates to that for sodium), we must suspend judgment whether or not the kinetics of penetration of the two ions differ fundamentally.

\section{REFERENCES}

Davson, H. (1955). J. Physiol. (Lond.), 129, 111. 203. Duke-Elder, S., MAURICF, D. M., Ross, E. J., and Woodin, A. M. (1949). Ibid., 108, and Matchett, P. A. (1953). Ibid., 122, 11.

Kinsey, V. E., and Grant, W. M. (1942). J. gen. Physiol., 26, 131.

and PALM, E. (1955). Arch. Ophthal. (Chicago), 53, 330.

Langham, M., and Davson, H. (1949). Biochem. J., 44, 467. 